\title{
Peritonitis outcomes in patients with HIV and end-stage renal failure on peritoneal dialysis: a prospective cohort study
}

\author{
Kwazi C. Z. Ndlovu ${ }^{1,2^{*}}$, Wilbert Sibanda ${ }^{3}$ and Alain Assounga ${ }^{1,2}$
}

\begin{abstract}
Background: Few studies have investigated the management of human immunodeficiency virus (HIV)-associated end-stage renal failure particularly in low-resource settings with limited access to renal replacement therapy. We aimed to evaluate the effects of HIV infection on continuous ambulatory peritoneal dialysis (CAPD)-associated peritonitis outcomes and technique failure in highly active antiretroviral therapy (HAART)-treated HIV-positive CAPD populations.
\end{abstract}

Methods: We conducted a single-center prospective cohort study of consecutive incident CAPD patients recruited from two hospitals in Durban, South Africa from September 2012-February 2015. Seventy HIV-negative and 70 HIV-positive end-stage renal failure patients were followed monthly for 18 months at a central renal clinic. Primary outcomes of peritonitis and catheter failure were assessed for the first 18 months of CAPD therapy. We assessed risk factors for peritonitis and catheter failure using Cox regression survival analysis.

Results: The HIV-positive cohort had a significantly increased rate of peritonitis compared to the HIV-negative cohort (1.86 vs. 0.76 episodes/person-years, respectively; hazard ratio [HR], 2.41; 95\% confidence interval [Cl], 1.69-3.45, $P<0.001)$. When the baseline CD4 count was below 200 cells $/ \mu \mathrm{L}$, the peritonitis rate rose to 3.69 episodes/person-years ( $\mathrm{HR}$ 4.54, 95\% Cl 2.35-8.76, $P<0.001$ ), while a baseline CD4 count above 350 cells/ $\mathrm{\mu L}$ was associated with a peritonitis rate of 1.60 episodes/person-years (HR 2.10, Cl 1.39-3.15, $P=0.001$ ). HIV was associated with increased hazards of peritonitis relapse ( $\mathrm{HR}, 3.88 ; \mathrm{Cl}, 1.37-10.94 ; P=0.010)$. Independent predictors associated with increased peritonitis risk were HIV (HR, 1.84; $\mathrm{Cl}, 1.07-3.16 ; P=0.027)$, diabetes (HR, 2.09; $\mathrm{Cl}, 1.09-4.03 ; P=0.027)$ and a baseline CD4 count $<200$ cells/ $\mu \mathrm{L}(\mathrm{HR}, 3.28 ; \mathrm{Cl}, 1.42-7.61 ; P=0.006)$. Catheter failure rates were 0.34 (HIV-positive cohort) and 0.24 (HIV-negative cohort) episodes/person-years (HR, 1.42; 95\% Cl, 0.73-2.73; $P=0.299)$. Peritonitis (HR, 14.47; Cl, 2.79-75. $00 ; P=0.001$ ), average hemoglobin concentrations ( $\mathrm{HR}, 0.75 ; \mathrm{Cl}, 0.59-0.95 ; P=0.016)$, and average serum $C$-reactive protein levels were independent predictors of catheter failure.

Conclusions: HIV infection in end-stage renal disease patients managed by CAPD was associated with increased peritonitis risk; however, HIV infection did not increase the risk for CAPD catheter failure rate at 18 months.

Keywords: Continuous ambulatory peritoneal dialysis (CAPD), End-stage renal disease (ESRD), HIV, Peritonitis, Infection, Technique failure, Catheter failure

\footnotetext{
* Correspondence: kczndlovu@gmail.com; ndlovuk@ukzn.ac.za

${ }^{1}$ Inkosi Albert Luthuli Central Hospital, Durban, South Africa

${ }^{2}$ Department of Nephrology, University of KwaZulu-Natal, P/Bag X7, Congella,

Durban 4013, South Africa

Full list of author information is available at the end of the article
} 


\section{Background}

Continuous ambulatory peritoneal dialysis (CAPD) is the dialysis modality of choice for many patients with end-stage renal disease (ESRD) and a cost-effective option easily implemented in low-resource settings [1-3]. However, peritonitis presents an ongoing challenge and is a major cause of technical failure [4-6], particularly under poor socioeconomic conditions and in immunocompromised patients [7, 8]. Considerable advancement has been made in CAPD management over the last decades leading to a substantial decrease in peritonitis rates, with as few as 1 case/51 patient-months reported by some authors [9]. However, peritonitis remains an important factor influencing CAPD-associated morbidity and mortality, and certain organisms, such as fungi and Gram-negative bacteria, are associated with worse outcomes [10-12]. Although reports are inconsistent, some of the factors associated with increased peritonitis risks are age, race, sex, comorbidities (diabetes, human immunodeficiency virus [HIV]), socioeconomic status, smoking, higher body mass index (BMI), malnutrition and chronic inflammation [4, 8, 13-16].

HIV infection presents a unique challenge in patients with ESRD managed with CAPD. As HIV impairs local host defense mechanisms [16], the risk of peritonitis in this population may be influenced by the adequacy of viral control and the patient's immunologic state [8, 17]. Furthermore, the protein and amino acid losses frequently observed in CAPD may aggravate the malnutrition and hypoalbuminemia common in HIV infection, which can further compound the risk of peritonitis [18-20]. The rates of non-communicable diseases such as chronic kidney diseases (CKD) among HIV-positive populations are expected to rise significantly, as highly active antiretroviral therapy (HAART) becomes widely accessible, and life expectancy improves [21]. However, in economically disadvantaged regions such as subSaharan Africa where the HIV population is disproportionately concentrated, only a small percentage of those in need are expected to have access to renal replacement therapy [21-23]. CAPD is a relatively inexpensive, easily learned, and readily implemented dialysis option that does not require complex equipment $[1-3,18]$. As such, it is particularly well suited as a home dialysis modality in regions where dialysis facilities are limited. However, peritonitis may complicate the use of CAPD in patients with ESRD and HIV. This study aimed to evaluate the effects of HIV infection on CAPD-associated peritonitis rates and outcomes, and to assess risk factors associated with the development of peritonitis and technique failure in HAART-treated HIV-positive CAPD populations.

\section{Methods}

The study protocol was approved by the University of KwaZulu-Natal Biomedical Research Ethics Committee (BE 187/11), and research was conducted in accordance with the principles of the Declaration of Helsinki. All participants provided written informed consent prior to study enrollment.

\section{Sites}

We recruited patients for a prospective cohort study from two hospitals in Durban, South Africa between September 2012 and February 2015. King Edward VIII Hospital (KEH) is a 799-bed regional referral center with limited specialist services. Inkosi Albert Luthuli Central Hospital (IALCH) is an 846-bed specialist referral hospital for KwaZulu-Natal province and covers a catchment area of approximately 10 million people. The renal unit based in IALCH offers CAPD (total patient population of 220), hemodialysis (150 patients), and transplantation services.

\section{Study population}

We enrolled $70 \mathrm{HIV}$-negative and $70 \mathrm{HIV}$-positive patients with end-stage renal failure who underwent dialysis with a newly inserted double-cuffed coiled Tenckhoff catheter at the two hospitals. Patients with incident CAPD aged 18-60 years were consecutively recruited soon after Tenckhoff insertion until each cohort reached the 70-patient target. Peritonitis rate differentials reported by previous similar studies were used to calculate the sample size required to achieve a power of $80 \%$ and an $\alpha$ error probability of 0.05 [8]. The HIV infection status was determined by two $4^{\text {th }}$ generation HIV enzymelinked immunosorbent assays (ELISA) performed by the South African National Health Laboratory Service (NHLS) before enrollment, screening for HIV performed using a HIV Ag/Ab Combo (CHIV) assay (ADVIA Centaur $^{\oplus}$ XP, Siemens Healthcare Diagnostics, Tarrytown, NY, USA) and confirmation using HIV Combi and HIV Combi PT assays (Cobas e601, Roche Diagnostics, Mannheim, Germany). HAART management was left to the discretion of the local clinic. Tenckhoff catheter insertion was performed by experienced surgeons by laparoscopy (66 HIV-negative and 35 HIV-positive patients) at IALCH, and by trained nephrologists percutaneously at KEH (4 HIV-negative and $20 \mathrm{HIV}$-positive patients) and IALCH (15 HIV-positive patients). All CAPD patients utilized Y-sets, twin-bag systems, and conventional peritoneal dialysis (PD) solutions (Dianeal $1.5,2.5$, or $4.25 \%$ dextrose, icodextrin, or amino acidbased solutions; Baxter Healthcare, Deerfield, IL, US). They were trained predominantly as outpatients by the same nursing team, and generally performed four exchanges per day. 


\section{Enrollment and follow-up}

On enrollment, demographic, clinical, and biochemical data were recorded. All patients were followed-up at a central renal clinic at IALCH monthly for 18 months or until the endpoints of catheter removal or death. At each follow-up, vital signs, clinical assessment, anthropometric measurements, and phlebotomy for biochemical tests were done by the research team, and details of peritonitis events and hospital admissions in the intervening period were recorded on predefined questionnaires. Laboratory tests for full blood count, C-reactive protein (CRP), and serum urea, creatinine, electrolytes, albumin, and ferritin were performed by the NHLS, and results were periodically retrieved from the IALCH electronic results database.

\section{Peritonitis episodes}

A peritonitis episode was defined as a clinical presentation with a cloudy effluent or abdominal pain associated with an effluent white blood cell count (WCC) of more than 100 cells/ $\mu \mathrm{L}$ or a positive PD effluent culture. The diagnosis of peritonitis was made by the CAPD nurse and the attending physician. The attending physician decided whether to manage the case on an inpatient or outpatient basis depending on the severity of the clinical presentation. All patients initially received intraperitoneal vancomycin and amikacin empirically, and further therapy was modified according to culture results. Treatment duration was typically two weeks unless extended to three weeks by the attending physician due to the cultured organism or response to treatment. Episodes of peritonitis were recorded on predefined questionnaires during monthly clinic visits along with the date of presentation, whether treated as inpatient or outpatient, presenting PD WCC, and the culture result retrieved from the hospital electronic record. PD effluent WCCs were manually assessed using a 40X microscope, and PD effluent culturing was performed by the NHLS microbiology department using standard culturing techniques.

Peritonitis-associated hospital admission was defined as an admission for which peritonitis was cited as one of the indications or where peritonitis was diagnosed during the admission. The hospitalization episodes were recorded on the predefined questionnaire with the date of admission and discharge, indications for, and outcome of the admission. Catheter removal occurring during a peritonitis-associated admission episode was recorded as being related to peritonitis.

Multiple peritonitis episodes were classified as relapsing if occurring within 4 weeks of completion of treatment for a prior episode with the same organism or one sterile episode, recurrent if occurring within 4 weeks of completion of treatment for a prior episode with a different organism, or repeat if occurring more than 4 weeks after completion of treatment for the prior episode [24].

\section{Endpoints}

All Tenckhoff catheters were removed at IALCH. The indications for removal and the corresponding date were recorded as study endpoints. Technique failure was defined as catheter removal due to catheter malfunction or infection. The in-hospital mortality dates at IALCH and certified causes of death were recorded. Deaths occurring outside IALCH were recorded as home deaths, and the corresponding details were obtained via telephone interviews with the participants' relatives.

\section{Statistical analysis}

Continuous variables are expressed as mean \pm standard deviation or medians (interquartile range [IQR]) and were compared using the Student's $t$-test or WilcoxonMann-Whitney test as appropriate. Proportions and categorical variables were compared using Pearson's chi-square test or Fisher's exact test as appropriate. Survival estimates were computed using the KaplanMeier method, and the log-rank test was used to compare survival curves. Univariate Cox regression survival analysis was used to estimate the association between HIV, associated subgroups, and various risk factors for outcome variables. Multivariable Cox regression analysis was used to identify independent predictors of survival. All analyses were performed using Stata Statistical Software, Release 13 (StataCorp, College Station, Texas, US). The level of significance was set at $P<0.05$.

\section{Results}

\section{Patient characteristics}

The mean patient age was $39.1 \pm 11.7$ (HIV-negative) and $37.0 \pm 9.4$ (HIV-positive) years with women accounting for 42.9 and $52.9 \%$ of the two cohorts, respectively. All patients (100\%) in the HIV-positive cohort were of African ethnicity compared to $84.3 \%$ in the HIVnegative cohort $(P=0.003)$. Fifty-one percent of HIVpositive patients were either newly diagnosed with HIV or had recently started HAART (less than six months before insertion of the Tenckhoff catheter). However, $57.1 \%$ of HIV-positive patients had a suppressed viral load ( $<150$ copies $/ \mathrm{mL}$, hospital laboratory assay limit) at the time of enrollment. While the median baseline viral load was 4230 copies/mL (IQR, 903-91,143) for patients with detectable viral loads, the median dropped below the detectable limit (IQR <150-2990) when including patients with undetectable viral loads. Twenty-one percent of HIV-positive patients had CD4 counts $>500$ cells $/ \mu \mathrm{L}, 20.0 \%$ had $<200$ cells $/ \mu \mathrm{L}$, and the remainder (58.6\%) had $200-500$ cells $/ \mu \mathrm{L}$. Other details of the study 
Table 1 Baseline characteristics

\begin{tabular}{|c|c|c|c|}
\hline Variable & $\begin{array}{l}\text { HIV-Negative } \\
(n=70)\end{array}$ & $\begin{array}{l}\text { HIV-Positive } \\
(n=70)\end{array}$ & $P$ value \\
\hline Age $($ mean $\pm S D)$ & $39.1 \pm 11.7$ & $37.0 \pm 9.4$ & $0.247^{a}$ \\
\hline Weight, kg (mean \pm SD) & $68.9 \pm 12.6$ & $66.1 \pm 13.7$ & $0.213^{\mathrm{a}}$ \\
\hline Body mass index (mean \pm SD) & $25.1 \pm 4.7$ & $24.5 \pm 5.4$ & $0.436^{\mathrm{a}}$ \\
\hline $\begin{array}{l}\text { Waist circumference, } \mathrm{cm} \\
\text { (mean } \pm \text { SD) }\end{array}$ & $90.5 \pm 10.9$ & $90.0 \pm 11.5$ & $0.822^{a}$ \\
\hline \multicolumn{4}{|l|}{ Sex } \\
\hline Female, $n(\%)$ & $30(42.9)$ & $37(52.9)$ & $0.236^{\mathrm{b}}$ \\
\hline \multicolumn{4}{|l|}{ Ethnicity } \\
\hline African, $n(\%)$ & $59(84.3)$ & $70(100.0)$ & $0.001^{c}$ \\
\hline Indian, n (\%) & $9(12.9)$ & $0(0.0)$ & \\
\hline Mixed ethnicity, $n(\%)$ & $2(2.9)$ & $0(0.0)$ & \\
\hline Hypertension, $n$ (\%) & $63(90.0)$ & $52(74.3)$ & $0.015^{b}$ \\
\hline Diabetes, n (\%) & $4(5.7)$ & $7(10.0)$ & $0.532^{c}$ \\
\hline SLE, $n(\%)$ & $4(5.7)$ & $1(1.4)$ & $0.366^{c}$ \\
\hline Hepatitis B, n (\%) & $7(10.0)$ & $8(12.1)$ & $0.737^{b}$ \\
\hline \multicolumn{4}{|l|}{ Primary residence } \\
\hline City, n (\%) & $14(20.0)$ & $6(8.6)$ & $0.113^{b}$ \\
\hline Township ${ }^{\mathrm{e}}, n(\%)$ & $30(42.8)$ & 39 (55.7) & \\
\hline Rural, $n(\%)$ & $23(32.9)$ & $24(34.3)$ & \\
\hline \multicolumn{4}{|l|}{ Education level } \\
\hline Primary school, $n(\%)$ & $15(21.4)$ & $13(18.6)$ & $0.710^{\mathrm{b}}$ \\
\hline High school, $n(\%)$ & $32(45.7)$ & $31(44.3)$ & \\
\hline Post-grade $12, n(\%)$ & $20(28.6)$ & $25(35.7)$ & \\
\hline \multicolumn{4}{|l|}{ Employment history } \\
\hline Unemployed, n (\%) & $50(71.4)$ & $53(75.7)$ & $0.766^{b}$ \\
\hline Employed, $n(\%)$ & $17(24.3)$ & $16(22.9)$ & \\
\hline Smoking (currently) & $4(5.71)$ & $7(10.0)$ & $0.532^{c}$ \\
\hline \multicolumn{4}{|c|}{ Tenckhoff catheter insertion method } \\
\hline Laparoscopic, n (\%) & $66(94.3)$ & $35(50.0)$ & $<0.001^{\mathrm{c}}$ \\
\hline Percutaneous, $n$ (\%) & $4(5.71)$ & $35(50.0)$ & \\
\hline Hemoglobin, $\mathrm{g} / \mathrm{dL}($ mean $\pm \mathrm{SD})$ & $9.60 \pm 2.01$ & $8.96 \pm 1.61$ & $0.041^{\mathrm{a}}$ \\
\hline Albumin, $g / L($ mean $\pm S D)$ & $35.3 \pm 6.7$ & $31.0 \pm 6.6$ & $<0.001^{\mathrm{a}}$ \\
\hline $\begin{array}{l}\mathrm{eGFR}, \mathrm{ml} / \mathrm{min} / 1.73 \mathrm{~m}^{2} \\
(\text { mean } \pm \mathrm{SD})\end{array}$ & $7.0 \pm 3.6$ & $7.1 \pm 4.6$ & $0.935^{a}$ \\
\hline $\begin{array}{l}\text { Creatinine }(\mu \mathrm{mol} / \mathrm{I}) \text {, } \\
\text { median (IQR) }\end{array}$ & $\begin{array}{l}736.5 \\
(542-974)\end{array}$ & $718(598-888)$ & $0.917^{d}$ \\
\hline CRP (mg/L), median (IQR) & $19(6-35)$ & $56.5(21-108)$ & $<0.001^{d}$ \\
\hline ESR (mm/hr), median (IQR) & $49(29-66)$ & $88(50-129)$ & $<0.001^{d}$ \\
\hline Ferritin $(\mu \mathrm{g} / \mathrm{L})$, median (IQR) & $\begin{array}{l}642 \\
(370-1049)\end{array}$ & $593(381-973)$ & $0.858^{d}$ \\
\hline
\end{tabular}

CD4 count

$\begin{array}{ll}\text { mean }(\text { cells } / \mu \mathrm{L} \pm \mathrm{SD}) & 380.7 \pm 235.4 \\ \text { CD4 }<200 \text { cells/ } \mu \mathrm{L}, n(\%) & 14.0(20.0) \\ \text { CD4 200-350 cells } / \mu \mathrm{L}, n(\%) & 20.0(28.6)\end{array}$

Table 1 Baseline characteristics (Continued)

\begin{tabular}{|c|c|}
\hline CD4 350-500 cells/ $\mu \mathrm{L}, n(\%)$ & $21.0(30.0)$ \\
\hline CD4 $\geq 500$ cells/ $\mu \mathrm{L}, n(\%)$ & $15.0(21.4)$ \\
\hline \multicolumn{2}{|l|}{ Viral load } \\
\hline Median, copies/mL (IQR) & $\begin{array}{l}4230 \\
(903-91143)\end{array}$ \\
\hline$<150$ copies/mL, $n(\%)$ & $40(57.1)$ \\
\hline 150-1000 copies/mL, n (\%) & $8(11.4)$ \\
\hline > 1000 copies/mL, n (\%) & $22(31.4)$ \\
\hline \multicolumn{2}{|l|}{ HAART history at enrollment } \\
\hline$<6$ months, $n(\%)$ & $36(51.4)$ \\
\hline 6-12 months, $n(\%)$ & $9(12.9)$ \\
\hline$>1$ year, $n(\%)$ & $25(35.7)$ \\
\hline \multicolumn{2}{|l|}{ HAART drug regimens } \\
\hline 3TC/EFV/ABC, $n(\%)$ & $59(84.3)$ \\
\hline 3TC/EFV/AZT, $n(\%)$ & $2(2.9)$ \\
\hline 3TC/EFV/D4T, n (\%) & $3(4.3)$ \\
\hline 3TC/NVP/ABC, $n(\%)$ & $3(4.3)$ \\
\hline 3TC/Alluvia/ABC, n (\%) & $1(1.43)$ \\
\hline 3TC/Aluvia/AZT, $n$ (\%) & $1(1.43)$ \\
\hline \multicolumn{2}{|c|}{ 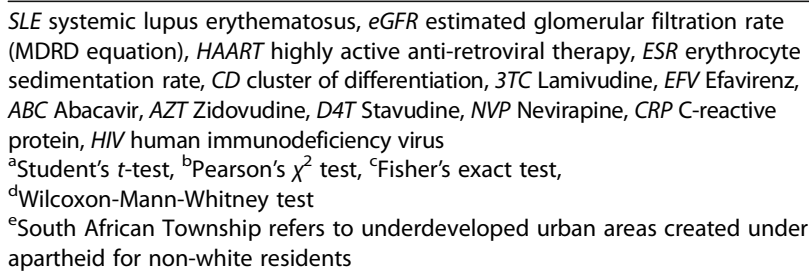 } \\
\hline
\end{tabular}

population are outlined in Table 1 and were previously described in our report on 1-year outcomes [25].

\section{Study end points}

After 18 months, 54.3\% (38/70) of the HIV-negative cohort and $28.6 \%(20 / 70)$ of the HIV-positive cohort were alive with a patent catheter $(P=0.002)$. Technique failure occurred in $24.3 \%(17 / 70)$ of the HIV-negative cohort and 27.1\% (19/70) of the HIV-positive cohort $(P=0.699)$, whereas $18.8 \%(13 / 70)$ and $40.0 \%$ (28/70), respectively, died $(P=0.005)$. One HIV-negative cohort participant and two HIV-positive participants had their Tenckhoff catheters removed due to improved renal function. One HIVnegative participant underwent kidney transplantation from a live related donor, and one HIV-positive participant left the study to undergo private hemodialysis and was lost to follow-up.

\section{Peritonitis episodes}

There were 54 peritonitis episodes observed in 44.3\% $(31 / 70)$ of the HIV-negative cohort and 94 episodes in $65.7 \%$ (46/70) of the HIV-positive cohort during the follow-up period $(P=0.011)$. Fifty-one percent $(36 / 70)$ of 
the HIV-positive cohort participants had one or more peritonitis episodes in the first 180 days following Tenckhoff catheter insertion compared to 24.3\% (17/ $70)$ in the HIV-negative cohort $(P=0.002)$. Nine percent $(5 / 54)$ of peritonitis episodes in the HIV-negative cohort and 13.8\% (13/94) in the HIV-positive cohort were relapse episodes $(P=0.602)$. Eleven percent $(6 / 54)$ of peritonitis episodes in the HIV-negative cohort and $7.4 \%$ (7/94) in the HIV-positive cohort were repeat episodes with the same organism $(P=0.448)$, while $18.5 \%$ $(10 / 54)$ in the HIV-negative cohort and $23.4 \%(22 / 94)$ in the HIV-positive cohort were repeat episodes with a different organism $(P=0.487)$ (Table 2).

The HIV-negative cohort had a higher proportion of Gram-negative peritonitis episodes $(44.4 \%, 24 / 54)$ compared to the HIV-positive cohort $(27.7 \%, 26 / 94)$ $(P=0.038)$. Culture-negative results were seen in $18.5 \%(10 / 54)$ of HIV-negative and $28.7 \%(27 / 94)$ of HIV-positive cohort episodes $(P=0.17)$ (Table 3$)$. The majority of the peritonitis episodes, $70.4 \%(38 / 54)$ in the HIV-negative cohort and $78.7 \%(74 / 94)$ in the HIV-positive cohort, were successfully treated without discontinuation of CAPD. Peritonitis resulted in catheter removal in $25.9 \%(14 / 54)$ of HIV-negative cases and $17.0 \%(16 / 94)$ of HIV-positive cases accounting for $82.4 \%(14 / 17)$ and $84.2 \%(16 / 19)$ of all technique failures in each cohort, respectively. A small proportion of peritonitis episodes $(3.7 \%(2 / 54)$ in the HIVnegative group and $4.3 \%(4 / 94)$ in the HIV-positive group) ended in mortality during observed admissions, accounting for $15.4 \%(2 / 13)$ and $14.3 \%(4 / 28)$ of all deaths in each cohort respectively (Table 2 ).

\section{Peritonitis rates and proportional hazard analysis}

Overall peritonitis rates, excluding relapse episodes, were 0.765 (HIV-negative cohort) and 1.855 (HIV-positive cohort) episodes/person-years (1/15.7 months and 1/6.5 months, respectively), with a Cox univariate proportional hazard ratio of 2.41 (95\% confidence interval (CI), 1.69-3.45, $P<0.001$ ) associated with HIV infection. Kaplan-Meier peritonitis-free survival rates were $6.0 \%$ (HIV-positive cohort) and 32.3\% (HIV-negative cohort) at 18 months $(P<0.001)$ (Fig. 1). When the baseline CD4 count was below 200 cells $/ \mu \mathrm{L}$, the peritonitis rate

Table 2 Peritonitis outcomes at 18 months

\begin{tabular}{|c|c|c|c|}
\hline & HIV-Negative & HIV-Positive & $P$ value \\
\hline Peritonitis episodes, $n$ ( $n$ excluding relapse) ${ }^{a}$ & $54(49)$ & $94(81)$ & $0.001^{b}$ \\
\hline Participants with a peritonitis episode at 180 days $^{\mathrm{e}}$ & $24.3 \%(17 / 70)$ & $51.4 \%(36 / 70)$ & 0.001 \\
\hline Participants with a peritonitis episode at 1 year $\mathrm{e}^{\mathrm{e}}$ & $38.6 \%(27 / 70)$ & $60.0 \%(42 / 70)$ & 0.011 \\
\hline Participants with a peritonitis episode at 18 months ${ }^{\mathrm{e}}$ & $44.3 \%(31 / 70)$ & $65.7 \%(46 / 70)$ & 0.011 \\
\hline \multicolumn{4}{|l|}{ Multiple peritonitis episodes } \\
\hline Time between peritonitis episodes (days), median (IQR) & $75(39-107)$ & $55.5(34.5-115.5)$ & $0.503^{d}$ \\
\hline Relapse & $9.2 \%(5 / 54)$ & $13.8 \%(13 / 94)$ & $0.602^{c}$ \\
\hline Recurrent & $3.7 \%(2 / 54)$ & $6.4 \%(6 / 94)$ & $0.711^{c}$ \\
\hline Repeat (Same organism) & $11.1 \%(6 / 54)$ & $7.4 \%(7 / 94)$ & $0.448^{\mathrm{b}}$ \\
\hline Repeat (Different organism) & $18.5 \%(10 / 54)$ & $23.4 \%(22 / 94)$ & $0.487^{b}$ \\
\hline \multicolumn{4}{|l|}{ Time - Tenckhoff catheter insertion to peritonitis episode } \\
\hline Median, days (IQR) & $184.5(98-370)$ & $144.5(63-296)$ & $0.124^{d}$ \\
\hline Within 180 days & $50.0 \%(27 / 54)$ & $56.4 \%(53 / 94)$ & $0.453^{\mathrm{b}}$ \\
\hline Between 180 - 365 days & $24.1 \%(13 / 54)$ & $27.7 \%(26 / 94)$ & $0.634^{\mathrm{b}}$ \\
\hline Between 365 - 550 days & $25.9 \%(14 / 54)$ & $16.0 \%(15 / 94)$ & $0.141^{b}$ \\
\hline 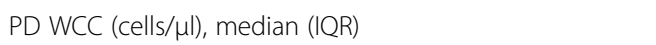 & $1073(360-2690)$ & $979(360-2370)$ & $0.927^{d}$ \\
\hline Outpatient treatment & $33.3 \%(18 / 54)$ & $40.4 \%(38 / 94)$ & $0.392^{b}$ \\
\hline Inpatient treatment & $66.7 \%(36 / 54)$ & $59.6 \%(56 / 94)$ & $0.392^{b}$ \\
\hline Inpatient stay (days), median (IQR) & $9(7-12)$ & $8(7-14.5)$ & $0.574^{d}$ \\
\hline \multicolumn{4}{|l|}{ Peritonitis episode outcomes } \\
\hline CAPD continuation & $70.4 \%(38 / 54)$ & $78.7 \%(74 / 94)$ & $0.254^{\mathrm{b}}$ \\
\hline Catheter removal & $25.9 \%(14 / 54)$ & $17.0 \%(16 / 94)$ & $0.195^{\mathrm{b}}$ \\
\hline Mortality & $3.7 \%(2 / 54)$ & $4.3 \%(4 / 94)$ & $1.000^{c}$ \\
\hline
\end{tabular}

$I Q R$ interquartile range, $P D$ peritoneal dialysis, WCC white blood cell count, CAPD continuous ambulatory peritoneal dialysis, HIV human immunodeficiency virus aPeritonitis episode count excluding peritonitis relapse' ${ }^{\text {b Pearson's }} X^{2}$ test, 'Fisher's exact test

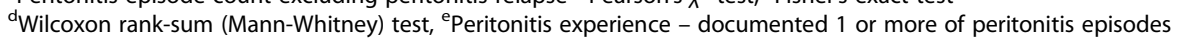


Table 3 Peritonitis episode culture results

\begin{tabular}{|c|c|c|c|}
\hline & HIV-Negative & HIV-Positive & $P$ value \\
\hline \multicolumn{4}{|l|}{ All peritonitis episodes } \\
\hline Gram-positive & $33.3 \%(18 / 54)$ & $36.2 \%(34 / 94)$ & $0.728^{a}$ \\
\hline Staphylococcus aureus & $16.7 \%(9 / 54)$ & $7.4 \%(7 / 94)$ & $0.082^{\mathrm{a}}$ \\
\hline Coagulase-negative staphylococcus & $16.7 \%(9 / 54)$ & $23.4 \%(22 / 94)$ & $0.332^{\mathrm{a}}$ \\
\hline Other gram-positive bacteria & $0.0 \%(0 / 54)$ & $5.3 \%(5 / 94)$ & $0.159^{b}$ \\
\hline Gram-negative & $44.4 \%(24 / 54)$ & $27.7 \%(26 / 94)$ & $0.038^{\mathrm{a}}$ \\
\hline Pseudomonas species & $13.0 \%(7 / 54)$ & $6.4 \%(6 / 94)$ & $0.173^{a}$ \\
\hline Klebsiella pneumoniae & $5.6 \%(3 / 54)$ & $4.3 \%(4 / 94)$ & $0.706^{\mathrm{b}}$ \\
\hline Acinetobacter species & $3.7 \%(2 / 54)$ & $10.6 \%(10 / 94)$ & $0.212^{\mathrm{b}}$ \\
\hline Other gram-negative bacteria & $22.2 \%(12 / 54)$ & $6.4 \%(6 / 94)$ & $0.005^{\mathrm{a}}$ \\
\hline Mixed organisms & $0.0 \%(0 / 54)$ & $1.1 \%(1 / 94)$ & $1.000^{\mathrm{b}}$ \\
\hline Fungal peritonitis & $3.7 \%(2 / 54)$ & $5.3 \%(5 / 94)$ & $1.000^{\mathrm{b}}$ \\
\hline Mycobacterium tuberculosis & $0.0 \%(0 / 54)$ & $1.1 \%(1 / 94)$ & $1.000^{\mathrm{b}}$ \\
\hline Culture-negative & $18.5 \%(10 / 54)$ & $28.7 \%(27 / 94)$ & $0.168^{\mathrm{a}}$ \\
\hline \multicolumn{4}{|l|}{ Peritonitis relapse episodes } \\
\hline Coagulase-negative staphylococcus & $1.8 \%(1 / 54)$ & $3.2 \%(3 / 94)$ & $1.000^{\mathrm{b}}$ \\
\hline Other gram-positive bacteria & $0.0 \%(0 / 54)$ & $1.1 \%(1 / 94)$ & $1.000^{\mathrm{b}}$ \\
\hline Pseudomonas species & $1.8 \%(1 / 54)$ & $3.2 \%(3 / 94)$ & $1.000^{\mathrm{b}}$ \\
\hline Other gram-negative bacteria & $1.8 \%(1 / 54)$ & $1.1 \%(1 / 94)$ & $1.000^{\mathrm{b}}$ \\
\hline Culture-negative & $3.7 \%(2 / 54)^{c}$ & $4.3 \%(4 / 94)^{d}$ & $1.000^{\mathrm{b}}$ \\
\hline Mycobacterium tuberculosis & $0.0 \%(0 / 54)$ & $1.1 \%(1 / 94)$ & $1.000^{\mathrm{b}}$ \\
\hline \multicolumn{4}{|l|}{ Peritonitis-associated catheter removal } \\
\hline Staphylococcus aureus & $1.8 \%(1 / 54)$ & 0 & $0.365^{\mathrm{b}}$ \\
\hline Pseudomonas species & $9.3 \%(5 / 54)$ & $1.1 \%(1 / 94)$ & $0.025^{b}$ \\
\hline Acinetobacter species & $1.8 \%(1 / 54)$ & $6.4 \%(6 / 94)$ & $0.423^{\mathrm{b}}$ \\
\hline Other gram-negative bacteria & $5.6 \%(3 / 54)$ & $1.1 \%(1 / 94)$ & $0.138^{b}$ \\
\hline Culture-negative & $3.7 \%(2 / 54)$ & $3.2 \%(3 / 94)$ & $1.000^{\mathrm{b}}$ \\
\hline Candida species & $3.7 \%(2 / 54)$ & $4.2 \%(4 / 94)$ & $1.000^{\mathrm{b}}$ \\
\hline Mycobacterium tuberculosis & 0 & $1.1 \%(1 / 94)^{\mathrm{e}}$ & $1.000^{\mathrm{b}}$ \\
\hline \multicolumn{4}{|l|}{ Peritonitis-associated mortality } \\
\hline Coagulase-negative staphylococcus & $1.8 \%(1 / 54)$ & $1.1 \%(1 / 94)$ & $1.000^{\mathrm{b}}$ \\
\hline Escherichia coli & $1.8 \%(1 / 54)$ & $1.1 \%(1 / 94)$ & $1.000^{\mathrm{b}}$ \\
\hline Fungal Peritonitis & 0 & $1.1 \%(1 / 94)$ & $1.000^{\mathrm{b}}$ \\
\hline Culture-negative & 0 & $1.1 \%(1 / 94)$ & $1.000^{\mathrm{b}}$ \\
\hline
\end{tabular}

HIV human immunodeficiency virus

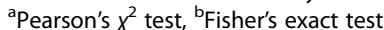

'One relapse episode first presented as Staphylococcus aureus peritonitis then relapsed as culture-negative peritonitis

${ }^{\mathrm{d}}$ Two relapse episodes first presented as Pseudomonas aeruginosa peritonitis then relapsed as culture-negative peritonitis

${ }^{e}$ First presented as culture-negative peritonitis then relapsed as Mycobacterium tuberculosis

rose to 3.690 episodes/person-years (HR 4.54, 95\% CI 2.35-8.76, $P<0.001$ ), while a baseline CD4 count above 350 cells $/ \mu \mathrm{L}$ was associated with a peritonitis rate of 1.599 episodes/person-years (HR 2.10, CI 1.39$3.15, P=0.001)$. The peritonitis relapse rate was 0.078 (HIV-negative cohort) and 0.298 (HIV-positive cohort) episodes/person-years (HR 3.88, CI 1.37-10.94, $P=$ 0.01) (Table 4).

On multivariable analysis, HIV (HR 1.84, 95\% CI 1.07-3.16, $P=0.03$ ), diabetes, and a baseline CD4 count less than 200 cells $/ \mu \mathrm{L}$ were found to be independent predictors of peritonitis (Table 5). 


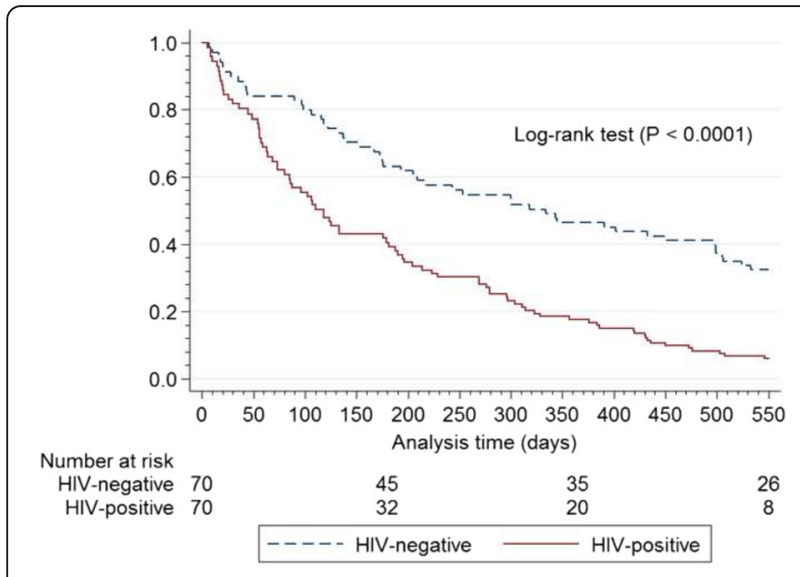

Fig. 1 Kaplan-Meier survival estimates for peritonitis episodes excluding relapses censored for mortality, catheter loss, and loss to follow-up HIV = human immunodeficiency virus

\section{Technique failure}

All-cause technique failure rates were 0.237 (HIV-negative cohort) and 0.338 (HIV-positive cohort) episodes/ person-years (HR 1.42, 95\% CI 0.73-2.73, $P=0.299$ ). Kaplan-Meier technique survival rates at 18 months censored for death, catheter removal not related to technique failure, and loss to follow-up were $71.4 \%$ (HIV-negative cohort) and 58.2\% (HIV-positive cohort), respectively $(P=0.295)$ (Fig. 2$)$. Fifty-three percent $(9 /$ 17) of technique failures in the HIV-negative cohort and $42.1 \%(8 / 19)$ in the HIV-positive cohort were due to gram-negative peritonitis episodes $(P=0.516)$. Fungal peritonitis was responsible for $11.8 \%(2 / 17)$ (HIV-negative cohort) and $21.0 \%(4 / 19)$ (HIV-positive cohort) of the technique failures $(P=0.662)$. Multivariable proportional hazard analysis identified peritonitis (HR 14.47, CI 2.7975.00, $P=0.001$ ), average hemoglobin concentration and average serum CRP level as independent predictors of technique failure (Table 5). Participants with one or more episodes of peritonitis during follow-up had an 18-month survival rate (Kaplan-Meier technique) of $47.5 \%$ compared to $93.9 \%$ for those who did not experience peritonitis $(P<0.0001)$ (Fig. 3$)$.

\section{Discussion}

This prospective cohort study evaluated the effect of HIV infection on CAPD-associated peritonitis outcomes in patients with ESRD requiring dialysis. At 18 months, HIV was associated with an increased risk (HR 2.41) of developing peritonitis with rates of 1.86 episodes/ person-years compared to 0.76 episodes/person-years for HIV-negative CAPD patients. Our HIV-negative peritonitis rate was higher than the target rate of $0.67 /$ year-atrisk advocated by the 2010 International Society for Peritoneal Dialysis (ISPD) guidelines, probably reflecting a higher intrinsic risk in our patient population which is predominantly impoverished with few available choices for alternative hemodialysis [24].

The few retrospective studies that have examined the outcomes of CAPD in HIV-infected patients have demonstrated improvements in survival and reductions in peritonitis rates associated with the use of HAART and advances in CAPD $[8,17,26]$. However, to our knowledge, our study is the first to prospectively evaluate the effects of HIV infection and duration of HAART on peritonitis outcomes among ESRD patients on CAPD. Our HIV-positive CAPD-associated peritonitis rate was much lower than the 3.9 episodes/patient-year reported over 20 years ago by Tebben et al. [17], reflecting a decreased risk associated with the greater availability of HAART over the years. Further, the authors reported a decreased peritonitis rate of 2.6 episodes/patient-year for HIV-positive patients using the Y-disconnect system, highlighting improved outcomes associated with technique enhancements. Khanna et al. [8] reported a lower peritonitis rate of 1.4 episodes/patient-year at the beginning of the HAART era; however, little information was provided on the characteristics of their HIVpositive CAPD population. Our state-sponsored renal replacement program practices a "PD first" policy directing that all dialysis-requiring ESRD patients be routinely started on CAPD. Limited hemodialysis slots are thereby reserved for those who fail CAPD or have medical contraindications to CAPD. This unselective policy determining our CAPD patient population along with the low educational levels and socioeconomic status of our patients (a majority being unemployed, living in impoverished areas, and not having completed grade 12) may have contributed to an increased intrinsic peritonitis risk [14, 27, 28].

Although HIV and diabetes were identified as independent predictors of poor peritonitis outcome, the immunologic state also modified the HIV-associated risk. A baseline CD4 count $<200$ cells $/ \mu \mathrm{L}$ increased the hazards for peritonitis more than 4-fold compared to HIV-negative CAPD patients (3.69 episodes/person-years, HR 4.54, $P<0.001)$. This probably reflects compromised host defense mechanisms against infectious organisms at lower CD4 counts. A baseline CD4 count above 350 cells $/ \mu \mathrm{L}$ was associated with a 2 -fold increased hazard for peritonitis (1.60 episodes/personyears, HR 2.10, $P=0.001$ ), further highlighting the inherent risk associated with HIV infection even with higher CD4 counts. The peritonitis risk was demonstrated to manifest early, as within 180 days following Tenckhoff catheter insertion half of the HIV-positive cohort had at least one documented episode of peritonitis. This risk was shown to persist, as demonstrated by the peritonitis-free survival rate of only $6.0 \%$ at 18 months. 
Table 4 Incidence rates and Cox proportional hazard univariate analysis

\begin{tabular}{|c|c|c|c|c|}
\hline Incidence rates per person-year & HIV-Negative & HIV-Positive & $\begin{array}{l}\text { Hazard ratio } \\
\text { (95\% Conf. Interval) }\end{array}$ & $P$ value \\
\hline All-cause peritonitis ${ }^{\mathrm{a}}$ & 0.765 & 1.855 & HR 2.41 (1.69-3.45) & $<0.001$ \\
\hline \multicolumn{5}{|l|}{ Baseline CD4 count } \\
\hline$<200$ cells/ $\mu \mathrm{L}$ & & 3.690 & HR 4.54 (2.35-8.76) & $<0.001$ \\
\hline 200-350 cells/uL & & 1.940 & HR 2.61 (1.60-4.24) & $<0.001$ \\
\hline$>350$ cells $/ \mu \mathrm{L}$ & & 1.599 & HR 2.10 (1.39-3.15) & 0.001 \\
\hline Gram-positive peritonitis & 0.262 & 0.675 & HR 2.59 (1.46-4.60) & 0.001 \\
\hline Gram-negative peritonitis & 0.353 & 0.512 & HR 1.40 (0.80-2.44) & 0.236 \\
\hline Culture-negative peritonitis & 0.150 & 0.560 & HR 3.64 (1.75-7.54) & 0.001 \\
\hline Fungal peritonitis & 0.028 & 0.089 & HR 3.25 (0.63-16.79) & 0.159 \\
\hline Peritonitis relapse & 0.078 & 0.298 & HR 3.88 (1.37-10.94) & 0.010 \\
\hline \multicolumn{5}{|l|}{ Baseline CD4 count } \\
\hline$<200$ cells $/ \mu \mathrm{L}$ & & 0.615 & HR 10.60 (1.95-57.56) & $0.006^{\mathrm{b}}$ \\
\hline 200-350 cells/ $\mu \mathrm{L}$ & & 0.698 & HR 8.82 (2.90-26.90) & $<0.001^{\mathrm{b}}$ \\
\hline$\geq 350$ cells $/ \mu \mathrm{L}$ & & 0.073 & HR 0.97 (0.19-5.00) & $0.969^{\mathrm{b}}$ \\
\hline Peritonitis recurrence & 0.031 & 0.137 & HR 4.62 (0.92-23.21) & 0.063 \\
\hline Peritonitis repeat (same organism) & 0.094 & 0.160 & HR $1.81(0.60-5.42)$ & 0.289 \\
\hline Peritonitis repeat (different organism) & 0.156 & 0.504 & HR 3.81 (1.80-8.09) & $<0.001$ \\
\hline Multiple peritonitis $^{\mathrm{a}}$ & 0.281 & 0.802 & HR $3.22(1.82-5.71)$ & $<0.001$ \\
\hline \multicolumn{5}{|l|}{ Baseline CD4 count } \\
\hline$<200$ cells/ $/ \mathrm{L}$ & & 1.230 & HR $8.41(2.71-26.08)$ & $<0.001^{\mathrm{b}}$ \\
\hline 200-350 cells/ $\mu \mathrm{L}$ & & 0.931 & HR 3.90 (1.86-8.18) & $<0.001^{\mathrm{b}}$ \\
\hline$>350$ cells $/ \mu \mathrm{L}$ & & 0.690 & HR $2.64(1.38-5.04)$ & $0.003^{b}$ \\
\hline Peritonitis hospital admissions & 0.815 & 1.814 & HR 2.19 (1.44-3.35) & $<0.001$ \\
\hline Peritonitis technique failure ${ }^{c}$ & 0.195 & 0.285 & HR 1.43 (0.69-2.93) & 0.335 \\
\hline All-cause technique failure ${ }^{d}$ & 0.237 & 0.338 & HR $1.42(0.73-2.73)$ & 0.299 \\
\hline Peritonitis mortality & 0.028 & 0.071 & HR 2.67 (0.49-14.60) & 0.258 \\
\hline All-cause mortality & 0.181 & 0.498 & HR $2.53(1.31-4.90)$ & 0.006 \\
\hline
\end{tabular}

HR hazard ratio, CD cluster of differentiation, HIV human immunodeficiency virus

${ }^{a}$ Excluding peritonitis relapse episodes; ${ }^{b}$ HIV-positive sub-groups compared to the HIV-negative cohort; ${ }^{c}$ Peritonitis technique failure - catheter removal due to peritonitis; ${ }^{d}$ Technique failure - catheter removal due to catheter malfunction or infection

The HIV-positive cohort showed an increased grampositive peritonitis rate compared to the HIV-negative cohort (0.68 vs. 0.26 episodes/person-years, HR 2.59, $P=0.001)$, possibly reflecting a greater susceptibility to touch contamination-related infection due to compromised local defense mechanisms contributing to the HIV-associated peritonitis risk. This finding suggests a role for a prophylactic antibiotic strategy, particularly in the first six months following catheter insertion when the peritonitis risk is highest and more so among patients with low CD4 counts. The HIV-positive cohort also had a significantly increased culture-negative peritonitis rate compared to the HIV-negative cohort (0.56 vs. 0.15 episodes/person-years, HR 3.64, $P=$ 0.001 ). Culture negative cases accounted for $28.7 \%$ of the HIV-positive cohort's total peritonitis episodes; this percentage is above the $20 \%$ recommended by the 2010 ISPD guidelines. This finding could indicate a higher prevalence of fastidious organisms and mycobacteria in this group $[29,30]$.

Peritonitis was shown to be the predominant cause of technique failure in both cohorts and was further identified as an independent predictor of this outcome. Although HIV was associated with an increased risk of peritonitis as well as an increased risk for subsequent episodes, it was not shown to significantly influence allcause technique failure rates (HR 1.42, $P=0.299$ ) or peritonitis-associated technique failure rates (HR 1.43, $P=0.335$ ). This inconsistency may be partially explained by the significantly higher proportion of gram-negative peritonitis episodes documented in the HIV-negative cohort (44.4 vs. $27.7 \%, P=0.038$ ); gram-negative organisms 
Table 5 Cox proportional hazard univariate and multivariate analyses: risk factors vs. peritonitis and technique failure

\begin{tabular}{|c|c|c|c|c|}
\hline \multirow[b]{2}{*}{ Variable } & \multicolumn{2}{|c|}{ Univariate Cox proportional hazards } & \multicolumn{2}{|c|}{ Multivariable Cox proportional hazards } \\
\hline & $\begin{array}{l}\text { Hazard ratio } \\
\text { (95\% Conf. Interval) }\end{array}$ & $P$ value & $\begin{array}{l}\text { Hazard ratio } \\
\text { (95\% Conf. Interval) }\end{array}$ & $P$ value \\
\hline \multicolumn{5}{|l|}{ Peritonitis $^{d}$} \\
\hline HIV & $2.41(1.69-3.45)$ & $<0.001$ & $1.84(1.07-3.16)$ & 0.027 \\
\hline Race & $0.19(0.06-0.57$ & 0.003 & $0.54(0.05-5.66)$ & 0.607 \\
\hline Catheter insertion method $^{a}$ & $1.86(1.28-2.71)$ & 0.001 & $0.63(0.28-1.42)$ & 0.269 \\
\hline Catheter insertion site $^{\mathrm{b}}$ & $2.20(1.45-3.33$ & $<0.001$ & $2.17(0.84-5.58)$ & 0.108 \\
\hline Diabetes & $2.22(1.35-3.66)$ & 0.002 & $2.09(1.09-4.03)$ & 0.027 \\
\hline BMl & $1.04(1.00-1.08$ & 0.033 & $1.01(0.95-1.09)$ & 0.720 \\
\hline Waist circumference & $1.02(1.00-1.04)$ & 0.017 & $1.03(0.99-1.06)$ & 0.151 \\
\hline Baseline hemoglobin & $0.85(0.77-0.94)$ & 0.002 & $1.02(0.88-1.19)$ & 0.786 \\
\hline Baseline albumin & $0.95(0.93-0.98)$ & 0.001 & $0.98(0.94-1.02)$ & 0.298 \\
\hline Baseline CRP & $1.00(1.00-1.01)$ & 0.004 & $1.00(1.00-1.00)$ & 0.652 \\
\hline \multicolumn{5}{|l|}{ Baseline CD4 count (cells/ $\mu \mathrm{L}$ ) } \\
\hline HIV-negative & Reference & & & \\
\hline CD4 $<200$ & $4.54(2.35-8.76)$ & $<0.001$ & $3.28(1.42-7.61)$ & 0.006 \\
\hline CD4 200-350 & $2.61(1.6-4.24)$ & $<0.001$ & $1.18(0.66-2.12)$ & 0.577 \\
\hline CD4 $\geq 350$ & $2.10(1.39-3.15)$ & $<0.001$ & 1.00 & \\
\hline \multicolumn{5}{|l|}{ Residence } \\
\hline City & Reference & & & \\
\hline Township ${ }^{c}$ & $5.94(2.17-16.25)$ & 0.001 & $5.56(0.82-37.50)$ & 0.078 \\
\hline Rural area & $6.07(2.19-16.8)$ & 0.001 & $4.68(0.70-31.07)$ & 0.110 \\
\hline \multicolumn{5}{|l|}{ Technique failure } \\
\hline HIV & $1.42(0.73-2.73)$ & 0.299 & $0.39(0.14-1.11)$ & 0.077 \\
\hline Peritonitis & $9.29(2.84-30.36)$ & $<0.001$ & $14.47(2.79-75.00)$ & 0.001 \\
\hline Catheter insertion site & $2.33(1.09-4.97)$ & 0.029 & $2.73(0.49-15.21)$ & 0.252 \\
\hline Catheter insertion method & $1.62(0.79-3.30)$ & 0.185 & $0.69(0.13-3.71)$ & 0.663 \\
\hline Average hemoglobin & $0.72(0.59-0.88)$ & 0.001 & $0.75(0.59-0.95)$ & 0.016 \\
\hline Average CRP & $1.02(1.01-1.02)$ & $<0.001$ & $1.02(1.01-1.03)$ & $<0.001$ \\
\hline
\end{tabular}

$B M I$ Body mass index, CD cluster of differentiation, CRP C-reactive protein, HIV human immunodeficiency virus

${ }^{a}$ Catheter insertion method- laparoscopic vs. percutaneous, ${ }^{b}$ Catheter insertion site - Inkosi Albert Luthuli Central Hospital vs King Edward VIII Hospital,

'South African Township refers to underdeveloped urban areas created under apartheid for non-white residents

${ }^{d}$ Adjusted for age, race, gender, smoking, diabetes, body mass index, waist circumference, baseline hemoglobin, baseline serum albumin, baseline C-reactive protein, primary residence, highest education level, employment, baseline CD4 count, Tenckhoff catheter insertion site, and Tenckhoff catheter insertion method (laparoscopic vs. percutaneous)

${ }^{\text {e}}$ Adjusted for HIV, peritonitis, age, gender, smoking, diabetes, body mass index, waist circumference, average hemoglobin, average C-reactive protein, average serum ferritin, primary residence, highest education level, employment, Tenckhoff catheter insertion site, and Tenckhoff catheter insertion method (laparoscopic vs. percutaneous)

were also the major causative organism group for technique failures in both cohorts $(52.9 \%$ and $42.1 \%$, respectively). It may be that HIV infection does not increase the risk of catheter-threatening peritonitis in the first 18 months following insertion but instead increases the risk for treatable peritonitis episodes. However, increased relapses and multiple episodes raise concern about the long-term risk of technique failure. Further, the disproportionately higher mortality rate in the HIV-positive cohort contributed to a dropout rate of $44.3 \%$ compared to $21.4 \%$ in the HIV- negative cohort; this may have introduced bias, resulting in a lower apparent rate of technique failure in the HIV-positive cohort.

The major limitation of our study is that it is a single-center observational study, which inherently limits causation inferences that can be drawn from observed associations. Statistical power, particularly relating to technique failure outcomes, was limited by the relatively small sample size and short follow-up period. The matching strategy of restricting the inclusion age to $18-60$ years may limit the generalizability of 


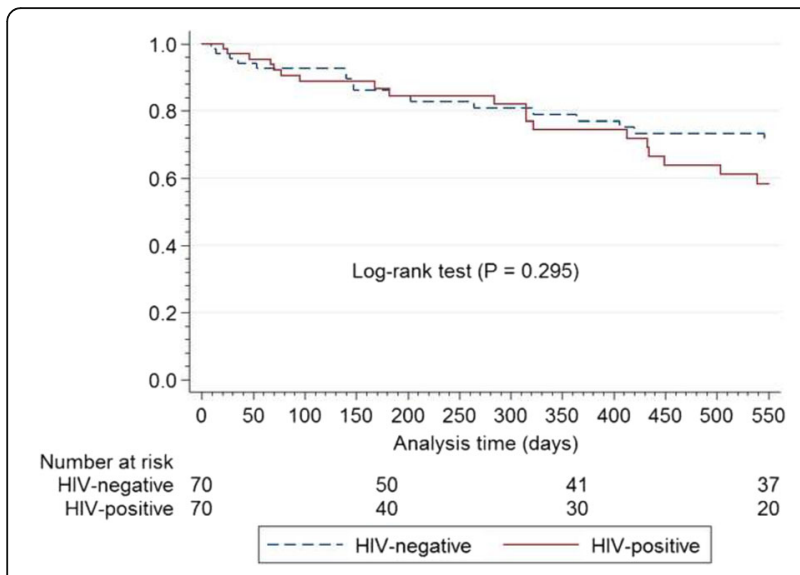

Fig. 2 Kaplan-Meier estimates for catheter patency according to HIV status censored for mortality, loss to follow-up, and catheter removal unrelated to technique failure HIV = human immunodeficiency virus

our results to only this age group. This matching strategy was employed to minimize age as a confounding factor, as HIV-positive CAPD populations are typically younger than their HIV-negative counterparts [8, 17]. More studies are needed to assess outcomes of renal replacement modalities in various HIV-positive ESRD populations.

\section{Conclusions}

Our study indicates that HIV infection can adversely influence CAPD-associated peritonitis rates, and this association is further modified by the immunological state of the infected patient. The peritonitis risk attributable to HIV infection manifests early in the course of CAPD treatment and increases the risk for subsequent episodes, but it was not shown to result in increased technique failure rates at 18 months. Early detection of

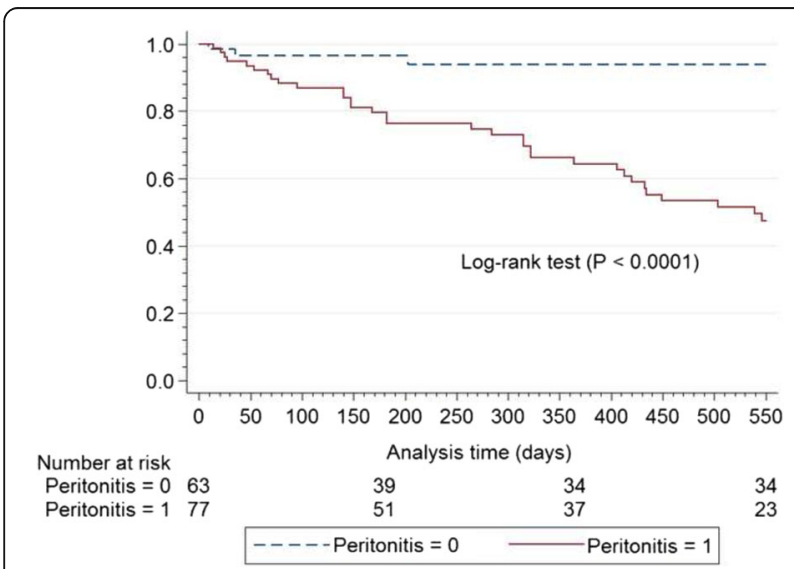

Fig. 3 Kaplan-Meier estimates for catheter patency according to peritonitis experience (1 or more peritonitis episodes during follow-up) censored for mortality, loss to follow-up, and catheter removal unrelated to catheter failure
CKD and HIV with the initiation of HAART before significant immunological compromise and careful management of comorbid conditions can help minimize the risk. Prophylactic antibiotics should be considered and investigated as possible strategies to help improve peritonitis outcomes.

\section{Abbreviations}

CAPD: Continuous ambulatory peritoneal dialysis; CD4: Cluster of differentiation 4; Cl: Confidence interval; CKD: Chronic kidney disease; CRP: C-reactive protein; ELISA: Enzyme-linked immunosorbent assay; ESRD: Endstage renal disease; HAART: Highly active antiretroviral therapy; HIV: Human immunodeficiency virus; HR: Hazard ratio; IALCH: Inkosi Albert Luthuli Central Hospital; IQR: Interquartile range; KEH: King Edward VIII Hospital; NHLS: South African National Health Laboratory Service; PD: Peritoneal dialysis; WCC: White blood cell count

\section{Acknowledgements}

The authors wish to thank Mr. James Bukenge Lukobeka, Sr. Lindiwe Beryl Mtambo, Sr. Busisiwe Msomi, Sr. Elizabeth Margaret Van Rooyen, Sr. Nontokozo Buthelezi, and all the staff of Inkosi Albert Luthuli Central Hospital Renal unit who helped with data collection.

\section{Funding}

This publication was made possible by funding from the International Society of Nephrology Clinical Research Program, Discovery Foundation Academic Fellowship Award, South African Medical Research Council Clinician Researcher Program, South African National Research Foundation Thuthuka Funding Instrument, University of Kwazulu-Natal College Of Health Sciences, and by grant number 5R24TW008863 from the Office of the U.S. Global AIDS Coordinator and the U. S. Department of Health and Human Services, National Institutes of Health (NIH OAR and NIH ORWH). Its contents are solely the responsibility of the authors and do not necessarily represent the official views of the government or funding organizations. Funders had no role in the design of the study and collection, analysis, and interpretation of data and in writing the manuscript.

\section{Availability of data and materials}

The datasets used and analyzed during the current study are available from the corresponding author on reasonable request.

\section{Authors' contributions}

Research idea and study design: KCZN, AA; data acquisition: KCZN; data analysis and interpretation: KCZN, WS, AA; statistical analysis: WS, KCZN; supervision and mentoring: AA. Each author contributed important intellectual content during manuscript drafting or revision and accepts accountability for the overall work by ensuring that questions pertaining to the accuracy or integrity of any portion of the work are appropriately investigated and resolved. All authors read and approved the final manuscript.

\section{Competing interests}

The authors declare that they have no competing interests.

\section{Consent for publication}

Not applicable.

\section{Ethics approval and consent to participate}

The study protocol was approved by the University of KwaZulu-Natal Biomedical Research Ethics Committee (BE 187/11), and research was conducted in accordance with the principles of the Declaration of Helsinki. All patients provided written informed consent prior to study enrollment.

\section{Author details}

${ }^{1}$ Inkosi Albert Luthuli Central Hospital, Durban, South Africa. ${ }^{2}$ Department of Nephrology, University of KwaZulu-Natal, P/Bag X7, Congella, Durban 4013, South Africa. ${ }^{3}$ School of Nursing and Public Health, University of KwaZulu-Natal, Durban, South Africa.

Received: 25 October 2016 Accepted: 31 January 2017 Published online: 03 February 2017 


\section{References}

1. Sennfalt K, Magnusson M, Carlsson P. Comparison of hemodialysis and peritoneal dialysis-a cost-utility analysis. Perit Dial Int. 2002;22:39-47.

2. Coentrao LA, Araujo CS, Ribeiro CA, Dias CC, Pestana MJ. Cost analysis of hemodialysis and peritoneal dialysis access in incident dialysis patients. Perit Dial Int. 2013:33:662-70.

3. Karopadi AN, Mason G, Rettore E, Ronco C. Cost of peritoneal dialysis and haemodialysis across the world. Nephrol Dial Transplant. 2013;28:2553-69.

4. Kumar VA, Sidell MA, Yang WT, Jones JP. Predictors of peritonitis, hospital days, and technique survival for peritoneal dialysis patients in a managed care setting. Perit Dial Int. 2014;34:171-8.

5. Troidle L, Gorban-Brennan N, Kliger A, Finkelstein FO. Continuous peritoneal dialysis-associated peritonitis: a review and current concepts. Semin Dial. 2003;16:428-37.

6. Brown MC, Simpson K, Kerssens JJ, Mactier RA, Scottish RR. Peritoneal dialysis-associated peritonitis rates and outcomes in a national cohort are not improving in the post-millennium (2000-2007). Perit Dial Int. 2011;31: 639-50

7. Vychytil A, Lorenz M, Schneider B, Hörl WH, Haag-Weber M. New strategies to prevent Staphylococcus aureus infections in peritoneal dialysis patients. J Am Soc Nephrol. 1998;9:669-76.

8. Khanna R, Tachopoulou OA, Fein PA, Chattopadhyay J, Avram MM. Survival experience of peritoneal dialysis patients with human immunodeficiency virus: a 17-year retrospective study. Adv Perit Dial. 2005;21:159-63.

9. Kopriva-Altfahrt G, König P, Mündle M, Prischl F, Roob JM, Wiesholzer M, et al. Exit-site care in Austrian peritoneal dialysis centers - a nationwide survey. Perit Dial Int. 2009;29:330-9.

10. Mujais S. Microbiology and outcomes of peritonitis in North America. Kidney Int Suppl. 2006;70:S55-62.

11. Ghali JR, Bannister KM, Brown FG, Rosman JB, Wiggins KJ, Johnson DW et al. Microbiology and outcomes of peritonitis in Australian peritoneal dialysis patients. Perit Dial Int. 2011:31:651-62.

12. Davenport A. Peritonitis remains the major clinical complication of peritoneal dialysis: the London, UK, peritonitis audit 2002-2003. Perit Dial Int. 2009;29:297-302

13. Pérez-Fontan M, Rodriguez-Carmona A, Garcia-Naveiro R, Rosales M, Villaverde P, Valdes F. Peritonitis-related mortality in patients undergoing chronic peritoneal dialysis. Perit Dial Int. 2005:25:274-84.

14. Martin LC, Caramori JC, Fernandes N, Divino-Filho JC, Pecoits-Filho R, Barretti $P$, et al. Geographic and educational factors and risk of the first peritonitis episode in Brazilian Peritoneal Dialysis study (BRAZPD) patients. Clin J Am Soc Nephrol. 2011;6:1944-51.

15. Chow KM, Szeto CC, Leung CB, Kwan BC, Law MC, Li PK. A risk analysis of continuous ambulatory peritoneal dialysis-related peritonitis. Perit Dial Int. 2005:25:374-9

16. Kotsanas D, Polkinghorne KR, Korman TM, Atkins RC, Brown F. Risk factors for peritoneal dialysis-related peritonitis: can we reduce the incidence and improve patient selection? Nephrology (Carlton). 2007;12:239-45.

17. Tebben JA, Rigsby MO, Selwyn PA, Brennan N, Kliger A, Finkelstein FO. Outcome of HIV infected patients on continuous ambulatory peritoneal dialysis. Kidney Int. 1993:44:191-8.

18. Gokal R, Mallick NP. Peritoneal dialysis. Lancet. 1999;353:823-8.

19. Prasad N, Gupta A, Sharma RK, Sinha A, Kumar R. Impact of nutritional status on peritonitis in CAPD patients. Perit Dial Int. 2007;27:42-7.

20. Leinig CE, Moraes T, Ribeiro S, Riella MC, Olandoski M, Martins C, et al. Predictive value of malnutrition markers for mortality in peritoneal dialysis patients. J Ren Nutr. 2011;21:176-83.

21. Stanifer JW, Jing B, Tolan S, Helmke N, Mukerjee R, Naicker S, et al. The epidemiology of chronic kidney disease in sub-Saharan Africa: a systematic review and meta-analysis. Lancet Glob Health. 2014;2:e174-81.

22. Naicker S. Burden of end-stage renal disease in sub-Saharan Africa. Clin Nephrol. 2010;74:S13-6.

23. Jha V, Garcia-Garcia G, Iseki K, Li Z, Naicker S, Plattner B, et al. Chronic kidney disease: global dimension and perspectives. Lancet. 2013;382:260-72.

24. Li PK, Szeto CC, Piraino B, Bernardini J, Figueiredo AE, Gupta A, et al. Peritoneal dialysis-related infections recommendations: 2010 update. Perit Dial Int. 2010;30:393-423.

25. Ndlovu KCZ, Assounga AG. Continuous ambulatory peritoneal dialysis in patients with HIV and renal failure. Perit Dial Int. 2016; In press.

26. Rivera Gorrin M, Merino Rivas JL, Alarcón Garcelán MC, Galeano Alvarez C, Manuel O, Teruel Briones $J$, et al. Outcome of HIV-infected patients of peritoneal dialysis: experience in a center and literature review. Nefrologia. 2008:28:505-10 [In Spanish].

27. Chern YB, Ho PS, Kuo LC, Chen JB. Lower education level is a major risk factor for peritonitis incidence in chronic peritoneal dialysis patients: a retrospective cohort study with 12-year follow-up. Perit Dial Int. 2013;33:552-8.

28. Wang Q, Hu KJ, Ren YP, Dong J, Han QF, Zhu TY, et al. The association of individual and regional socioeconomic status on initial peritonitis and outcomes in peritoneal dialysis patients: a propensity score-matched cohort study. Perit Dial Int. 2016;36:395-401.

29. Boyd MA, Laurens MB, Fiorella PD, Mendley SR. Peritonitis and technique failure caused by Roseomonas mucosa in an adolescent infected with HIV on continuous cycling peritoneal dialysis. J Clin Microbiol. 2012;50:3801-4.

30. Sadjadi SA, Obedoza P, Annamarju P. Moraxella Catarrhalis peritonitis. Am J Case Rep. 2012;13:19-21.

\section{Submit your next manuscript to BioMed Central and we will help you at every step:}

- We accept pre-submission inquiries

- Our selector tool helps you to find the most relevant journal

- We provide round the clock customer support

- Convenient online submission

- Thorough peer review

- Inclusion in PubMed and all major indexing services

- Maximum visibility for your research

Submit your manuscript at www.biomedcentral.com/submit 